\title{
Renal interstitial mast cell counts differ across classes of proliferative lupus nephritis
}

\author{
Karolina Kaczmarczyk ${ }^{1}$, Joanna Kosalka ${ }^{2}$, Jerzy Soja ${ }^{3}$, Marek Kuzniewski ${ }^{4}$ \\ Jacek Musial'2, Krzysztof Okon'1,
}

${ }^{1}$ Department of Pathomorphology, Collegium Medicum, Jagiellonian University, Cracow, Poland ${ }^{2}$ Department of Allergy and Immunology, Collegium Medicum, Jagiellonian University, Cracow, Poland ${ }^{3}$ Department of Pulmonology, Collegium Medicum, Jagiellonian University, Cracow, Poland ${ }^{4}$ Department of Nephrology, Collegium Medicum, Jagiellonian University, Cracow, Poland

\begin{abstract}
Systemic lupus erythematosus frequently involves the kidneys leading to significant morbidity and mortality. It is classified according to glomerular involvement pattern but tubulointerstitial lesions are also important for progression and prognosis, as seen in other kidney glomerular diseases. One of the cell types which participate in this process are mast cells. The aim of the study was to analyze the counts of tryptase-positive and chymase-positive mast cells in lupus nephritis classes II, III and IV. Material consisted of 42 renal biopsies from patients with lupus nephritis; 11 class II, 9 class III and 22 class IV. Chymase- and tryptase-containing cells were stained by immunohistochemistry and counted microscopically. Mean count of chymase-positive mast cells was 9.8/10 high power fields (hpf) for the whole group, 4.66 for class II, 11.89 for class III, and 11.51 for class IV. The mean count of tryptase-positive cells was 18.6/10 hpf for the whole group, 7.65 for class II, 25.57 for class III, and 21.23 for class IV. The differences between lupus nephritis classes were significant both for chymase- and tryptase-positive cells. Tryptase- but not chymase-positive cell counts showed a correlation with the creatinine level $(\mathrm{R}=0.35)$. These results suggest that mast cells are involved to a different degree in the pathogenesis of lupus nephritis depending on the class of the disease. (Folia Histochemica et Cytobiologica 2014, Vol. 52, No. 3, 218-224)
\end{abstract}

Key words: SLE; lupus nephritis; mast cell; chymase; tryptase; IHC

\section{Introduction}

Systemic lupus erythematosus (SLE) is an autoimmune disease involving several organs and systems, most prevalent in young to middle-aged women. Its prevalence worldwide varies from 40/100,000 in Northern Europeans to 100/100,000 in Africans and 150/100,000 in Brazilians; exact epidemiologic data from Poland is lacking, however, the frequency of SLE appears to be increasing [1-3]. 5-year and 10-year mortality in long-term studies is about $10 \%$ and $30 \%$ respectively,

Correspondence address: K. Okon, M.D., Ph.D.

Chair of Pathomorphology, Jagiellonian University,

Collegium Medicum

Grzegorzecka St. 16, 31-531 Krakow, Poland

e-mail: mpokon@cyf-kr.edu.pl with the main causes of death being infections, cardiovascular disease and direct organ damage [2-4]. Involvement of the kidney is frequent; lesions of particular importance are glomerulonephritis and the resulting tubulointerstitial changes. For prognostic and therapeutic purposes, glomerular lesions in SLE are classified by the International Society of Nephrology/Renal Pathology Society (ISN/RPS) system or the older World Health Organization (WHO) system [5]. It is important to note that renal involvement is the most frequent SLE organ damage-related cause of death [4].

Lupus nephritis (LN) results from the activity of multiple biologic factors like autoantibodies, inflammatory cells, growth factors, chemokines, cytokines, reactive oxygen and nitrogen species. Similarly to other forms of glomerulonephritis, glomerular inflammation leads to tubular epithelial cell activation, 


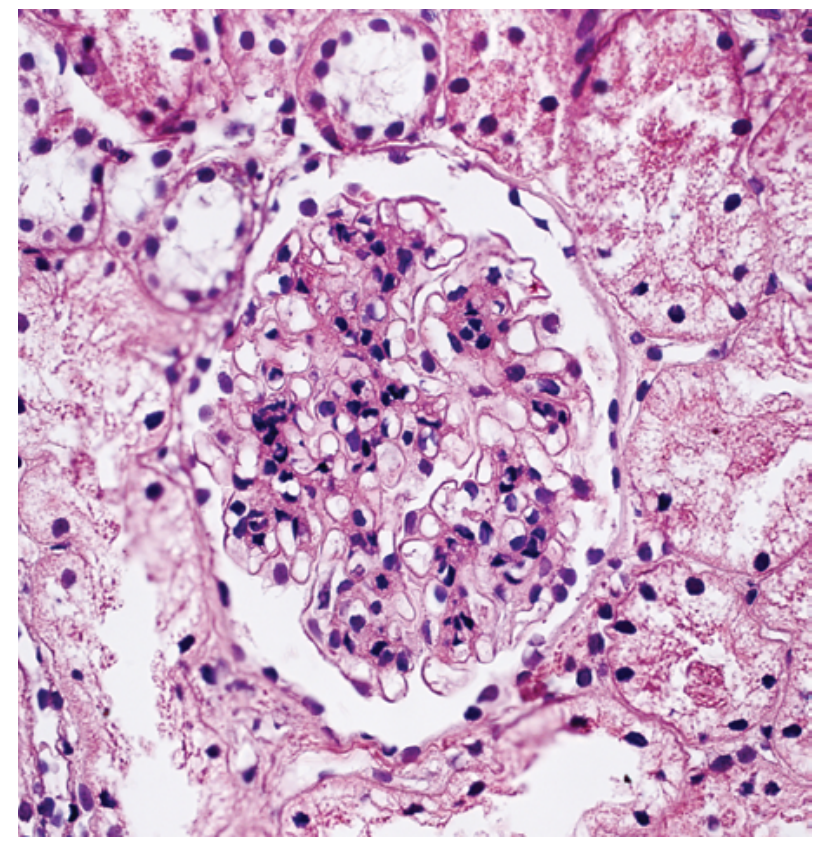

Figure 1. Glomerulus with only mild mesangial proliferation, consistent with lupus nephritis class II. Hematoxylin and eosin $(\mathrm{H} \& \mathrm{E})$ staining, original magnification $\times 400$

interstitial inflammation and interstitial fibrosis, which is ultimately responsible for renal failure [6-8]. One of the cell types present in chronically inflamed renal interstitium is the mast cell (MC). It has been shown that mast cells actively participate in interstitial inflammation and contribute to renal fibrosis [8-10].

Human mast cells are characterized by the presence of granules which contain two proteases: tryptase and chymase. Since only a proportion of mast cells is positive for chymase, two classes of mast cells, chymase-positive and chymase-negative, may be distinguished [11]. In cases of inflammation or injury, chymase is released into the interstitium and by activating latent transforming growth factor $-\beta$ (TGF- $\beta$ ), is implicated in tissue remodeling. The number of mast cells correlates with the severity of tubulointerstitial disease: chymase-positive mast cells correlate with the severity of interstitial fibrosis and intra-renal vascular resistance [9]. Mast cell tryptase may also be involved in the development of interstitial fibrosis [10].

The aim of the study was to analyze the counts of tryptase-positive and chymase-positive mast cells in lupus nephritis and compare these counts between ISN/RPS classes II, III and IV. We analyzed tubulointerstitial infiltration of chymase- and tryptase-positive mast cells in patients with lupus nephritis class II, III and IV and examined the relationship between lupus classification, cell infiltration and renal function.

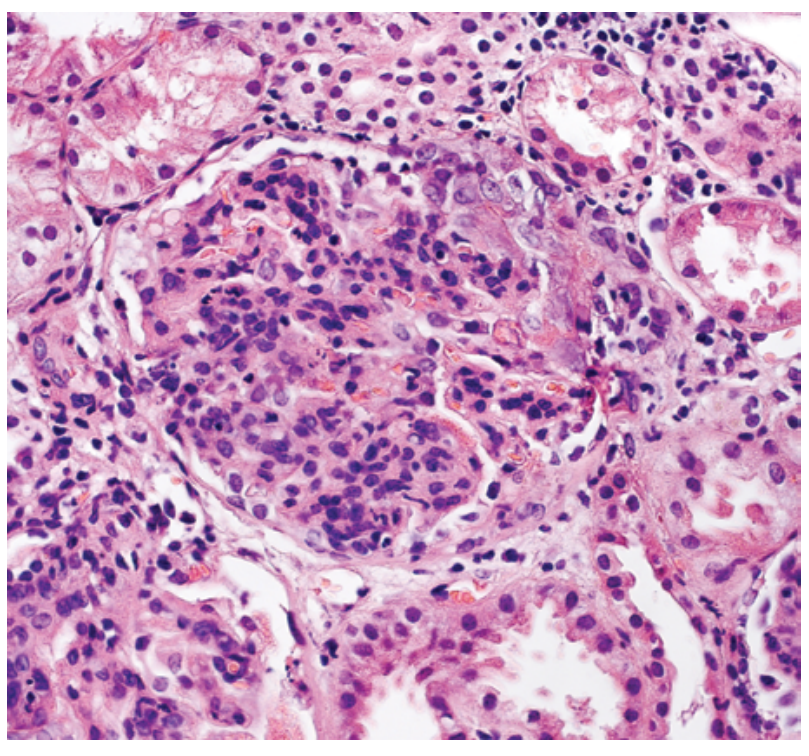

Figure 2. Extensive mesangial proliferation with increased lobulation may represent class III or class IV lesions, depending on the number of glomeruli involved $(<50 \%$ v. $>50 \%)$. H \& E staining, original magnification $\times 400$

\section{Material and methods}

Patients. The material consisted of representative renal biopsies from 42 patients with lupus nephritis ( 35 females and 7 males) retrieved from the archives of the Department of Pathomorphology (Collegium Medicum, Jagiellonian University, Cracow). Eleven patients were classified as lupus nephritis class II (Figure 1), 9 patients as class III, 22 patients as class IV (Figure 2).

Histology and histomorphology. The material was fixed in formalin, processed by routine method and embedded in paraffin. From the paraffin blocks, $4 \mu \mathrm{m}$ sections were cut and stained. For histologic classification, sections were stained by standard hematoxylin and eosin, periodic acid-Shiff, Massons' trichrome, Congo red, and Jones' silver methods. Only representative biopsies were used for this study. The cases were classified according to ISN/RPS criteria, and the activity and chronicity indexes as included in ISN/RPS system were calculated [5]. For the present study, only cases belonging to class II, III and IV were chosen; the more detailed sub-classification was disregarded for the purpose of statistical analysis.

Primary anti-tryptase antibody (1:100; Leica Biosystems $\mathrm{GmbH}$, Wetzlar, Germany) and anti-chymase antibody (1:100; Abcam, Cambridge, UK) were used. The immunohistochemistry was done by routine manual method. The Lab Vision detection system (Thermo Fisher Scientific, Waltham, MA, USA) was used. 3-amino-9-ethylcarbasole 


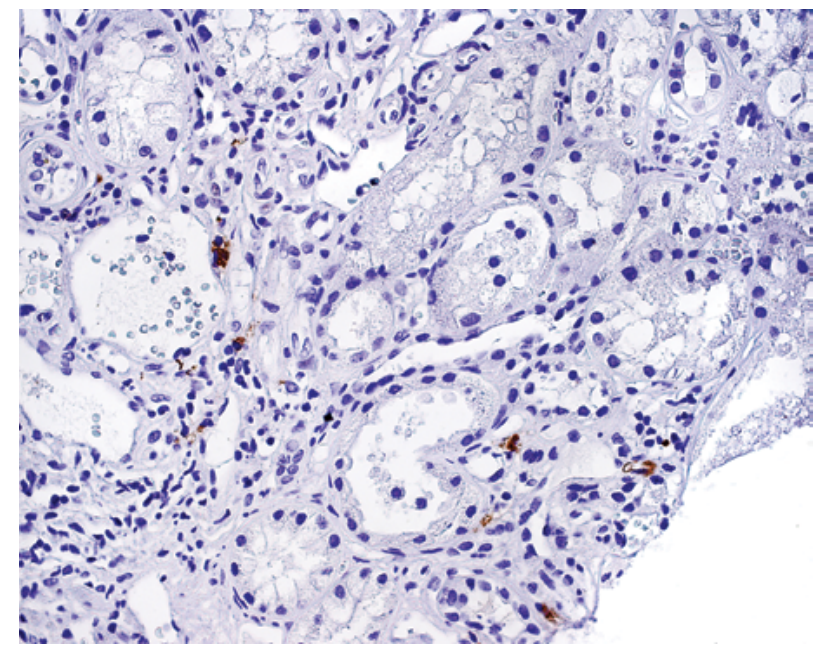

Figure 3. Chymase-positive cells in renal interstitium. Immunohistochemical staining was performed as described in Methods, original magnification $\times 400$

was used as the chromogen. The slides were counterstained with Mayer hematoxylin (Thermo Fisher Scientific) and coverslipped. The immunostained slides (Figures 3 and 4) were examined on Olympus CH20 microscope (Olympus, Tokyo, Japan) equipped with a $40 \times$ lens; the number of immunopositive cells, as well as number of fields of view were documented. The results were expressed as a sum of positive cells from 10 high power fields (hpf), while the area of one hpf was $0.159 \mathrm{~mm}^{2}$. The person performing the assessment was not aware of the diagnoses or clinical data. The values of serum creatinine and daily proteinuria were obtained from the patients' records.

For the assessment of relative interstitial volume (RIV), Massons' trichrome stained sections were used with a Zeiss Standard 25 (Zeiss GmbH, Oberkochen, Germany) microscope equipped with a 121-point rectangular eyepiece grid (Pyser-SGI Ltd., Edenbridge, UK) and a $40 \times$ lens. The well-preserved area of the renal cortex was scanned, noting both the number of fields of view and number of grid cross sections hitting the renal interstitium. The vessels larger than arterioles and glomeruli were disregarded. The result was expressed as the ratio of the points sampled in the interstitium to the total number of points sampled in the renal cortex.

Statistical methods. The statistical analysis was performed with Statistica 10 (StatSoft Inc., Tulsa, OK, USA). Kruskal-Wallis ANOVA and Mann-Whitney U tests were used, when appropriate. Correlations were measured by Spearman's correlation coefficient. The significance level was set at 0.05 .

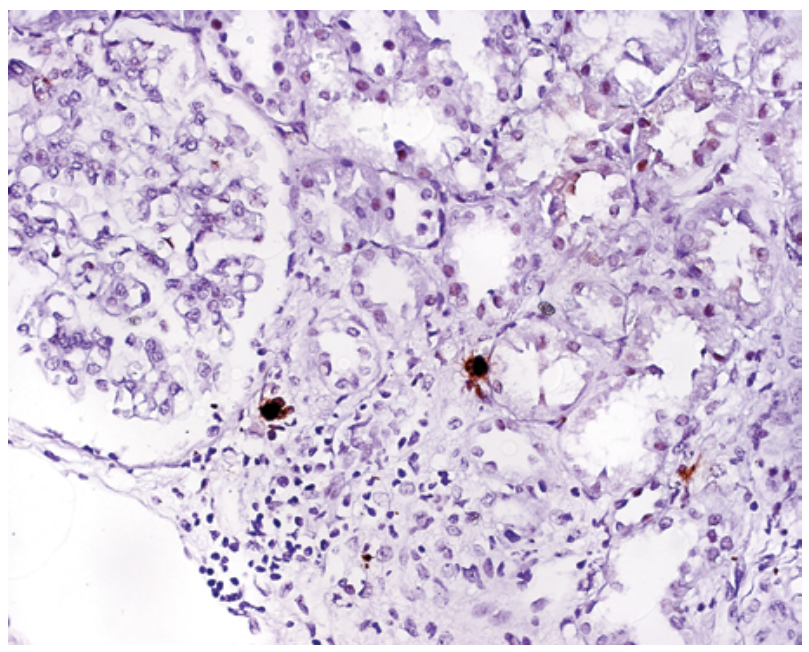

Figure 4. Tryptase-positive cells in renal interstitium. Immunohistochemistry, original magnification $\times 400$

\section{Results}

\section{Clinical characteristics of patients}

Mean age of all patients was $33.9 \pm 9.5$ years (all values expressed as arithmetic mean $\pm \mathrm{SD}$ ), range $19-56$ years. For class II the mean age was 38.5 \pm 14.3 , in class III $-36.0 \pm 10.0$, and in class IV it was $31.9 \pm 8.3$ years. Mean age was $34.2 \pm 10.5$ years for females and $33.3 \pm 11.7$ years for males. The differences between patient's mean age in the classes or gender were statistically non-significant.

Activity index determined according to ISN/RPS criteria [5] ranged from 1 to 14 (median 4), as expected, shown significant differences between the main ISN/RPS classes (median 1 for class II, 4 for class III and 7 for class IV, $p<0.001$ ). Chronicity index score determined according to ISN/RPS criteria [5] ranged from 0 to 8 (median 2), presented only slight and non-significant differences between ISN/RPS classes (median 1 for class II, 3 for class III and 2 for class IV). Mean creatinine serum concentration was 85.3 $\pm 38.1 \mu \mathrm{mol} / \mathrm{l}$, range 40 to $192 \mu \mathrm{mol} / \mathrm{l}$. In lupus nephritis class II mean creatinine level was 74.6 $\pm 24.1 \mu \mathrm{mol} / \mathrm{l}$, in class III $-86.5 \pm 34.2 \mu \mathrm{mol} / \mathrm{l}$, and in class IV $-90.3 \pm 43.9 \mu \mathrm{mol} / \mathrm{l}$. Mean daily proteinuria level was $3.5 \pm 3.0 \mathrm{~g} / \mathrm{d}$, range 0 to $11 \mathrm{~g} / \mathrm{d}$. In lupus nephritis class II mean daily proteinuria was $2.73 \pm 3.0 \mathrm{~g} / \mathrm{d}$, in class III $-2.62 \pm 1.4 \mathrm{~g} / \mathrm{d}$, and in class IV $-4.31 \pm 3.5 \mathrm{~g} / \mathrm{d}$. The differences between the lupus nephritis classes were statistically non-significant. 


\section{Histomorphometric evaluation of mast cells in the renal interstitium of lupus nephritis patients}

In all lupus nephritis patients the mean relative interstitial volume (RIV) was $0.18 \pm 0.09$, range 0.06 to 0.4 . In class II of lupus nephritis mean RIV was 0.14 , in class III -0.19 , and in class IV -0.20 (the differences between groups were non-significant).

The mean count of chymase-positive mast cells (MCs) was $9.8 \pm 8.6$ per 10 high power fields, while the mean count for tryptase-positive MCs was 18.6 \pm 18.4 . The average ratio of chymase-positive to tryptase-positive cells count was $0.77 \pm 0.71$.

Table 1 presents the values for each lupus nephritis class. The differences between chymase-positive and tryptase-positive cells counts were statistically significant $(\mathrm{p}<0.035$ and $\mathrm{p}<0.016$, respectively; Figures 5 and 6). On post-hoc analysis the differences in chymase-positive MCs were found between class II and IV, whereas the number of tryptase-positive MCs differed between class II and III and class II and IV.
The differences in the ratio of chymase-positive to tryptase-positive cells between lupus nephritis classes were not significant; however, there was a significant inverse correlation between the ratio of chymase-positive to tryptase-positive cells and patients' age $(\mathrm{R}=-0.36 ; \mathrm{p}<0.025)$. Chymase-positive $\mathrm{MC}$ counts and tryptase-positive MC counts were strongly correlated $(\mathrm{R}=0.67)$. Both chymase- and tryptase-positive $\mathrm{MC}$ counts correlated with disease activity index $(R=0.31$ and $R=0.46$, respectively) and a chronicity index $(\mathrm{R}=0.36$ and $\mathrm{R}=0.48$, respectively). Both chymase-positive MC counts and tryptase-positive MC counts correlated with RIV $(\mathrm{R}=0.35$ and $\mathrm{R}=0.40$, respectively). The tryptase-positive MC count showed a positive correlation with creatinine level $(\mathrm{R}=0.33)$ and proteinuria $(\mathrm{R}=0.31)$. The chymase-positive MC count showed a weak correlation with proteinuria level $(R=0.20)$ and RIV showed a weak correlation with creatinine serum concentration $(R=0.25)$ and patients' age $(\mathrm{R}=0.18)$. The disease activity index correlated with proteinuria $(\mathrm{R}=0.41)$.

Table 1. Mast cell counts in lupus nephritis classes as defined by International Society of Nephrology/Renal Pathology Society (ISN/RPS)

\begin{tabular}{|c|c|c|c|c|c|c|c|c|c|c|c|c|}
\hline \multirow{2}{*}{$\begin{array}{l}\text { ISN/RPS } \\
\text { class }\end{array}$} & \multicolumn{4}{|c|}{ Tryptase-positive } & \multicolumn{4}{|c|}{ Chymase-positive } & \multicolumn{4}{|c|}{ Chymase-/tryptase-positive ratio } \\
\hline & Mean & SD & Min & Max & Mean & SD & Min & Max & Mean & SD & Min & Max \\
\hline II (11) & 7.65 & 6.19 & 1 & 18 & 4.66 & 4.10 & 1 & 13 & 0.94 & 1.06 & 0.20 & 3.33 \\
\hline III (9) & $25.57^{*}$ & 21.17 & 4 & 68 & 11.89 & 11.22 & 1 & 33 & 0.49 & 0.37 & 0.13 & 1.42 \\
\hline IV (22) & $21.23^{*}$ & 19.46 & 2 & 79 & $11.51^{* *}$ & 8.33 & 3 & 30 & 0.80 & 0.59 & 0.05 & 2.50 \\
\hline
\end{tabular}

Number of patients is given in parenthesis. Mast cell counts were measured as described in Methods. SD — standard deviation; *, **significantly different from class II, $\mathrm{p}<0.05$ and $\mathrm{p}=0.01$, respectively; Kruskal-Wallis ANOVA

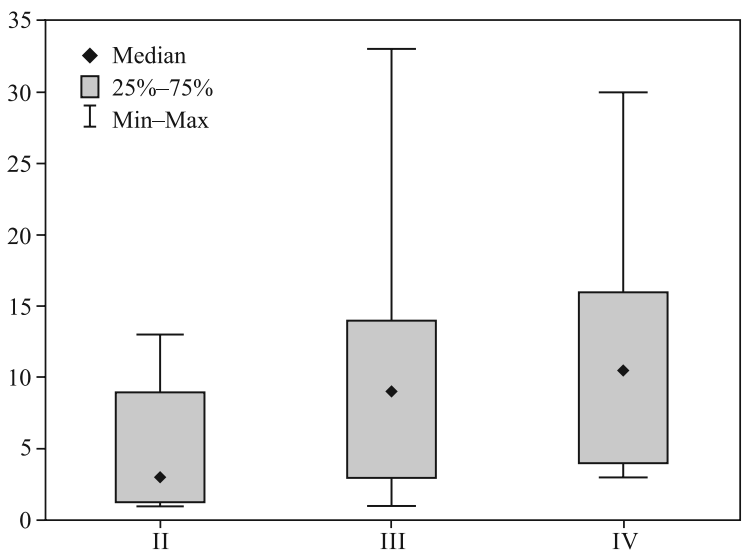

Figure 5. Number of chymase-positive cells by lupus nephritis ISN/RPS classes (II-IV). Y axis: number of cells per 10 high power fields

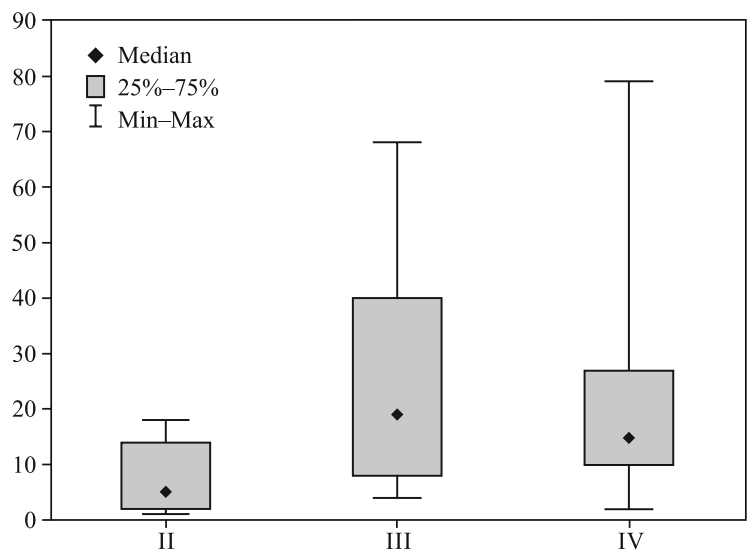

Figure 6. Number of tryptase-positive cells per high power field by lupus nephritis ISN/RPS classes (II-IV). Y axis: number of cells per 10 high power fields 


\section{Discussion}

As indicated above, lupus nephritis is an important cause of morbidity and mortality in patients with SLE [4]. The exact pathogenesis of lupus as well as lupus nephritis is yet to be definitely determined. The main mechanism responsible for organ injury, including kidney disease, is immune complex deposition. These immune complexes, which may consist of antinuclear antibodies and nuclear material, deposit in the tissues activating complement and interacting with complement receptors. Impaired immune complex clearing is thought to be one of the major pathogenetic mechanisms in SLE. In the kidney, the immune complexes are deposited primarily under the endothelium and in mesangium, leading to activation of mesangial cells and podocytes, which secrete a number of inflammatory mediators. This results in interstitial inflammation and, finally, renal interstitial fibrosis. Tubulointerstitial damage plays a key role in glomerulonephritis, including lupus nephritis; in most cases the decrease in glomerular filtration rate correlates more with interstitial kidney damage than with glomerular fibrosis [2]. Some authors have suggested that various forms of lupus nephritis may differ in their immunopathogenesis; only global forms would be related to classic immune complex deposition [12]. Renal interstitial infiltrates in SLE are very common, especially in more active (class III and IV) forms of the disease. These infiltrates are composed of a heterogeneous population of cells interacting with each other. Specifically T cells, B cells, macrophages and dendritic cells (DCs) play an important role in the development of lupus nephritis and kidney damage $[13,14]$ with each cell type possessing distinctive functions. Periglomerular inflammation caused by glomerular antigen-reactive $\mathrm{T}$ cells in the renal cortex leads to increased macrophage infiltration. Macrophages are most likely attracted to the renal interstitium by $T$ cell -derived signals [14]. B cells produce autoantibodies responsible for the tissue damage and may play a role as potential antigen presenting cells and regulators for the purpose of T cell [15], macrophage and DCs migration and activation [16]. Dendritic cells produce several pro-inflammatory and pro-fibrotic factors [17]. They enhance the presentation of auto-antigens and the induction of autoimmunity [18]. Additionally, they promote the expansion of $\mathrm{T}$ and $\mathrm{B}$ cells which leads to tissue damage [19].

Mast cells are also present in the inflammatory infiltrate, though in far lower number. Usually they may be visualized only by immunohistochemistry, thus their role in the pathogenesis of lupus nephritis was neglected. They show proinflammatory actions, secreting a great number of factors and taking part in many processes such as angiogenesis, bone remodeling, atherosclerosis, reactions to neoplasms and wound healing [20]. They are involved in chronic inflammation and renal fibrosis similar to tubulointerstitial damage which occurs during glomerulonephritis. Mast cells are rarely seen in healthy kidney, but their number increases in the kidney interstitium during disease; this can be seen in diabetic nephropathy, acute renal allografts rejection, IgA nephropathy, amyloidosis, renovascular ischemia, reflux nephropathy, polycystic kidney disease and drug-induced nephropathy [11, 21-24]. They were found in renal cortical tubulointerstitium, the periglomerular and perivascular areas, but not in glomeruli $[8,11,21]$. The number or mast cells parallels the tubulointerstitial injury and decline of renal function, measured by serum creatinine concentration [21].

There are some studies concerning quantification of mast cells in lupus nephritis, yet, little is known about the relative importance of mast cell populations, as well as their role in specific types of nephritis. Hiromura et al. analyzed both primary glomerulonephritis and kidney involvement in systemic processes including lupus [21]. They showed a significant correlation between the number of mast cells and the serum creatinine concentration at the time of biopsy but found no correlation between the number of mast cells and daily urinary protein excretion; this was true for all but crescentic glomerulonephritis. Similarly, in the present study the overall number of mast cells correlated with creatinine level. Unlike Hiromura et al., we found a significant correlation between the number of mast cells and proteinuria. The differences between studies may be due to different selection of cases, as we limited our material to class II, III and IV. Unfortunately, Hiromura et al. failed to report the details of lupus nephritis group [21]. Ravinal et al. [25] showed a correlation of tryptase-positive mast cells with tubulointerstitial $\alpha$-smooth muscle actin expression in class $V$ lupus nephritis, but not in the other classes. Furthermore, no correlations between the number of MCs and lupus outcome or interstitial TGF- $\beta 1$ expression were observed [25]. Danilewicz et al. analyzed similar material, and showed that the number of interstitial tryptase-positive cells was significantly increased in class $\mathrm{V}$ lupus nephritis as compared to idiopathic membranous glomerulopathy [26]. In both idiopathic and lupus-related disease, there were significant positive correlations between interstitial tryptase-positive MCs number and interstitial expansion, as well as serum creatinine level, which suggests possible relationship between mast cells and the development of interstitial fibrosis [26]. 
Our and other authors' results $[25,26]$ suggest that mast cells contribute to renal damage in different lupus nephritis classes. In our study, we were able to show significant differences between the individual classes of lupus nephritis which represents a novel finding, as yet not described in the literature.

The role of mast cells in other kinds of renal disease has been better appreciated. Danilewicz et al. [27] found a significant increase of mast cells in mesangiocapillary glomerulonephritis. In their material, MC count correlated with the degree of interstitial fibrosis, macrophage count, interstitial myofibroblasts and renal function parameters [27]. In an another study [28] the same group analyzed paired biopsies of patients with idiopathic mesangioproliferative glomerulonephritis. They found a significant increase in mast cell counts along with interstitial fibrosis, macrophage count and interstitial myofibroblasts, but not in T or B lymphocytes' number. Surprisingly, this interstitial remodeling was accompanied by reduction of proteinuria. Okon et al. [11] analyzed both primary glomerulonephritis and diabetic nephropathy and found a significant correlation between mast cell count, interstitial fibrosis and renal functional parameters. Highest MC counts were present in diabetic nephropathy, but an increase was also present in primary glomerulonephritis. Unlike in the present study, chymase-positive cells number showed stronger correlation with renal function than tryptase-positive cells [11]. Papadimitriou et al. analyzed the role of mast cells on renal allografts, and noticed a significant correlation between mast cell number and the degree of interstitial fibrosis but not with the activity of neither cellular nor humoral rejection [29]. On the other hand, some studies suggest that mast cells may act as a protective factor in renal disease. It has been hypothesized that one of the protective factors could be mast cell chymase [30]. Even better established is the role of chymase as an alternative angiotensin convertase; locally generated angiotensin may increase proteinuria as well as systemic blood pressure [31]. Our results suggest that chymase is generated in lupus nephritis, yet its influence on renal function is weaker than in other renal disease, such as diabetic nephropathy [11]. Interestingly, we found that the proportion of mast cells expressing chymase decreases with age; this feature was not observed in other diseases and by other studies.

In conclusion, for the first time we have shown that mast cell counts differ between classes of lupus nephritis. This could suggest that the role of these cells may differ across different classes of lupus nephritis.

\section{Acknowledgments}

The authors wish to thank Mr. Ian B. Perera for assistance with editing in English and Mr. Krzysztof Skomski for preparing the microphotographs.

\section{References}

1. Rahman A, Isenberg DA. Mechanisms of disease: Systemic lupus erythematosus. N Engl J Med. 2008;358:929-939.

2. Tsokos GC. Mechanisms of disease. Systemic lupus erythematosus. N Engl J Med. 2011;365:2110-2121.

3. Ruiz-Irastorza G, Khamashta MA, Castellino G et al. Systemic lupus erythematosus. Lancet 2001;357:1027-1032.

4. Trager J, Ward MM. Mortality and causes of death in systemic lupus erythematosus. Curr Opin Rheumatol. 2001;13: 345-351.

5. Weening JJ, D'Agati VD, Schwartz MM et al. The classification of glomerulonephritis in systemic lupus erythematosus revisited. Kidney Int. 2004;65:521-530.

6. Okon K. Tubulo-interstitial lesions in glomerulopathy. I. Pathogenesis. Pol J Pathol. 2003;54:87-94.

7. Okon K. Tubulo-interstitial changes in glomerulopathy. II. Prognostic significance. Pol J Pathol. 2003;54:163-169.

8. Holdsworth SR, Summers SA. Role of mast cells in progressive renal diseases. J Am Soc Nephrol. 2008;19:2254-2261.

9. Wasse H, Naqvi N, Husain A. Impact of mast cell chymase on renal disease progression. Curr Hypertens Rev. 2012;8:15-23.

10. Kondo S, Kagami S, Kido $\mathrm{H}$ et al. Role of mast cell tryptase in renal interstitial fibrosis. J Am Soc Nephrol. 2001;12:1668-1676.

11. Okon K, Stachura J. Increased mast cell density in renal interstitium is correlated with relative interstitial volume, serum creatinine and urea especially in diabetic nephropathy but also in primary glomerulonephritis. Pol J Pathol. 2007;58:193-197.

12. Hill GS, Delahousse M, Nochy D et al. Class IV-S versus class IV-G lupus nephritis: Clinical and morphologic differences suggesting different pathogenesis. Kidney Int. 2005;68: 2288-2297.

13. Bagavant H, Fu SM. Pathogenesis of kidney disease in systemic lupus erythematosus. Curr Opin Rheumatol. 2009;21: 489-494.

14. Tucci M, Stucci S, Strippoli S et al. Cytokine overproduction, T-cell activation, and defective T-regulatory functions promote nephritis in systemic lupus erythematosus. J Biomed Biotechnol. 2010;2010:457146.

15. Steinmetz OM, Velden J, Kneissler U et al. Analysis and classification of B-cell infiltrates in lupus and ANCA-associated nephritis. Kidney Int. 2008;74:448-457.

16. Clatworthy MR, Smith KG. B cells in glomerulonephritis: focus on lupus nephritis. Semin Immunopathol. 2007;29:337-353.

17. Fiore N, Castellano G, Blasi A et al. Immature myeloid and plasmacytoid dendritic cells infiltrate renal tubulointerstitium in patients with lupus nephritis. Mol Immunol. 2008;45:259-265.

18. Castellano G, Trouw LA, Fiore $\mathrm{N}$ et al. Infiltrating dendritic cells contribute to local synthesis of $\mathrm{C} 1 \mathrm{q}$ in murine and human lupus nephritis. Mol Immunol. 2010;47:2129-2137.

19. Teichmann LL, Ols ML, Kashgarian M et al. Dendritic cells in lupus are not required for activation of $\mathrm{T}$ and $\mathrm{B}$ cells but promote their expansion, resulting in tissue damage. Immunity 2010;33:967-978.

20. Dyduch G, Kaczmarczyk K, Okon K. Mast cells and cancer: enemies or allies? Pol J Pathol. 2012;63:1-7.

21. Hiromura K, Kurosawa M, Yano S et al. Tubulointerstitial mast cell infiltration in glomerulonephritis. Am J Kidney Dis. 1998;32:593-599. 
22. Ehara T, Shigematsu H. Contribution of mast cells to the tubulointerstitial lesions in iga nephritis. Kidney Int 1998;54:1675-1683.

23. Goto E, Honjo S, Yamashita $\mathrm{H}$ et al. Mast cells in human allografted kidney, correlation with interstitial fibrosis. Clin Transplant. 2002;16 (suppl 8):7-11.

24. McPherson EA, Luo Z, Brown RA et al. Chymaselike angiotensin II-generatingactivity in end-stage human autosomal dominant polycystic kidney disease. J Am Soc Nephrol. 2004;15:493-500.

25. Ravinal RC, Costa RS, Coimbra TM et al. Mast cells, TGF-beta1 and myofibroblasts expression in lupus nephritis outcome. Lupus 2005;14:814-821

26. Danilewicz M, Wagrowska-Danilewicz M. Quantitative analysis of interstitial mast cells in lupus and non-lupus membranous glomerulopathy. Pol J Pathol. 2001;52: 211-217.
27. Danilewicz M, Wagrowska-Danilewicz M. Quantitative analysis of the interstitial mast cells in idiopathic mesangiocapillary glomerulonephritis type I. Nefrologia 2001;21:253-259.

28. Danilewicz M, Wagrowska-Danilewicz M. Immunohistochemical analysis of the interstitial mast cells in rebiopsied patients with idiopathic mesangial proliferative glomerulonephritis. Pol J Pathol. 2005;56:63-68.

29. Papadimitriou JC, Drachenberg CB, Ramos E et al. Mast cell quantitation in renal transplant biopsy specimens as a potential marker for the cumulative burden of tissue injury. Transplant Proc. 2013;45:1469-1471.

30. Beghdadi W, Madjene LC, Claver J et al. Mast cell chymase protects against renal fibrosis in murine unilateral ureteral obstruction. Kidney Int. 2013;84:317-326.

31. Lu XF, Roksnoer LCW, Danser AHJ. The intrarenal renin -angiotensin system: does it exist? Implications from a recent study in renal angiotensin-converting enzyme knockout mice. Nephrol Dial Transplant. 2013;28:2977-2982.

Submitted: 27 March, 2014

Accepted after reviews: 21 July, 2014 